\title{
A theoretical study of the optical absorption band shape for xenon hexafluoride
}

\author{
William J. Ehlhardt and Lawrence L. Lohr, Jr. \\ Department of Chemistry, University of Michigan, Ann Arbor, Michigan 48109 \\ (Received 23 November 1976) \\ The classical Franck-Condon approximation is used together with the Monte Carlo integration technique \\ to calculate the optical absorption band shape arising in xenon hexafluoride from the pseudo-Jahn-Teller \\ active $t_{1 u}$ bending mode. The potential energy function for this mode has the Devonshire form for the \\ hindered rotational motion of a diatomic molecule in a cubic site and is characterized by three parameters. \\ Results are presented using values of these parameters as determined by Pitzer and Bernstein for the ${ }^{1} A_{18}$ \\ electronic ground state and as estimated by us from the crystal-field model of Wang and Lohr for the ${ }^{1} T_{1 u}$ \\ and ${ }^{3} T_{1 u}$ electronic excited states.
}

\section{INTRODUCTION}

It is now reasonably well established that the $\mathrm{XeF}_{6}$ molecule possesses a structure differing from that of an octahedral molecule primarily by a displacement along a $t_{1 u}$ bending coordinate. The resulting structure is nonrigid and can undergo a pseudorotational motion through eight equivalent structures of $C_{3 v}$ symmetry. This motion is thus in an octuple-well potential identical in form to the Devonshire potential ${ }^{1}$ for the hindered rotational motion of a diatomic molecule in a cubic symmetry environment. The form of this potential is given by

$$
\begin{aligned}
V(x, y, z)= & -a\left(x^{2}+y^{2}+z^{2}\right)+b\left(x^{4}+y^{4}+z^{4}\right) \\
& +(2 b-c)\left(x^{2} y^{2}+x^{2} z^{2}+y^{2} z^{2}\right),
\end{aligned}
$$

where $x, y$, and $z$ are the Cartesian components of the $t_{1 u}$ mode, or

$$
\begin{aligned}
V(r, \theta, \phi)= & -a r^{2}+b r^{4} \\
& -c r^{4}\left[\sin ^{4} \theta\left(\sin ^{2} \phi \cos ^{2} \phi-1\right)+\sin ^{2} \theta\right],
\end{aligned}
$$

where $r, \theta$ and $\phi$ are the corresponding spherical polar coordinates. The evidence in support of these conclusions comes mainly from the gas-phase electron diffraction studies by Gavin and Bartell, ${ }^{2,3}$ but is supported by the successful efforts of Pitzer and Bernstein ${ }^{4}$ to fit the calorimetrically determined entropy data ${ }^{5}$ and other observed properties using a model based on pseudoJahn-Teller considerations. Additional support is given by the results of the crystal-field model study by Wang and Lohr. ${ }^{6-8}$ For reasonable values of the electronic parameters they found a substantial distortion away from an octahedral structure, but no evidence for thermally accessible spin triplet states as necessitated by the electronic isomerism model which Goodman ${ }^{9}$ proposed to account for the electron diffraction data and the ir, Raman, and uv spectral results of Claassen, Goodman, and Kim. ${ }^{10}$ No evidence for the triplet states was found in the synchrotron radiation absorption studies by Nielsen, Hänsel, and Schwarz, ${ }^{11}$ so that at this writing it would appear that pseudorotation rather than electronic isomerism is the correct explanation for the unusual properties of the $\mathrm{XeF}_{6}$ gaseous molecule.

The uv absorption spectrum of $\mathrm{XeF}_{6}$ strongly resembles that of other hexahalo complexes of ions with $(n s)^{2}$ ground-state configurations. Figure 1 compares the gaseous $\mathrm{XeF}_{6}$ spectrum ${ }^{10}$ at $298^{\circ} \mathrm{K}$ and a pressure of 1-3 mm to that of the $\mathrm{TeCl}_{6}^{-2}$ complex ${ }^{12}$ in the solid $\left[n-\mathrm{Bu}_{4} \mathrm{~N}\right]_{2} \mathrm{TeCl}_{6}$ at 18 and $300^{\circ} \mathrm{K}$. It is reasonable to associate the bands at $25000 \mathrm{~cm}^{-1}$ for $\mathrm{TeCl}_{6}^{-2}$ and $30000 \mathrm{~cm}^{-1}$ for $\mathrm{XeF}_{6}$ with the ${ }^{1} A_{1 g}-{ }^{3} T_{1 u}$ transition (symmetry notation for the group $O_{h}$ used for convenience) and the bands at $34000 \mathrm{~cm}^{-1}$ for $\mathrm{TeCl}_{6}^{-2}$ and $42000 \mathrm{~cm}^{-1}$ for $\mathrm{XeF}_{6}$ with the ${ }^{1} A_{1 \varepsilon}-{ }^{1} T_{1 u}$ transition. However, an interesting feature of the $\mathrm{XeF}_{6}$ spectrum is the long "tail" of the first absorption band, with this tail not only extending well into the visible (to about $22000 \mathrm{~cm}^{-1}$ ) but also showing a marked increase in absorbance upon increasing the sample temperature from $298^{\circ} \mathrm{K}$ to $363^{\circ} \mathrm{K}$. By contrast the absorbance decreases with increasing temperature in the range $30000-33000 \mathrm{~cm}^{-1}$. These features were interpreted by Claassen, Goodman, and $\mathrm{Kim}^{10}$ using the electronic isomerism model, ${ }^{\theta}$ which leads to spectral assignments different from those given above.

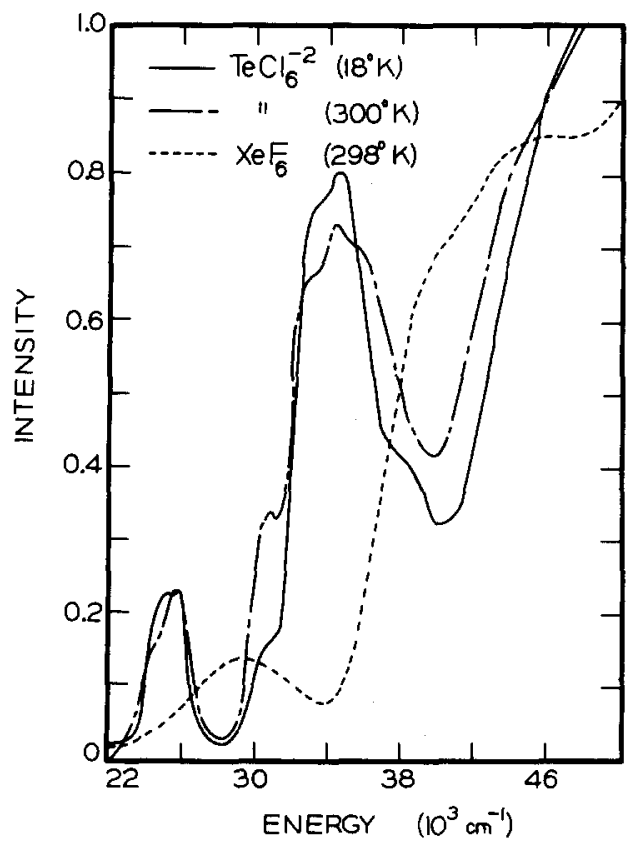

FIG. 1. Adaptations of the absorption spectra of solid $\left[\mathrm{Bu}_{4} \mathrm{~N}\right]_{2}$ $\mathrm{TeCl}_{6}$ at $18^{\circ} \mathrm{K}(-)$ and $300^{\circ} \mathrm{K}(-\infty-\infty)$, Ref. 12, and of gaseous $\mathrm{XeF}_{6}$ at $298^{\circ} \mathrm{K}(---)$, Ref. 10. The intensity scale is arbitrary, so no meaningful intensity comparison can be made between $\mathrm{TeCl}_{6}^{-2}$ and $\mathrm{XeF}_{6}$. 
Wang and Lohr ${ }^{8}$ calculated vibration energy levels and wavefunctions from their computer potential energy surface and used the results to calculate the absorption band shape as a function of temperature. Their procedure involved the expansion of the vibrational wavefunctions in a one-dimensional harmonic oscillator (HO) basis and is thus practical only for special cases of the Devonshire potential $(1,2)$ for which the threedimensional quantum mechanical problem reduces to one dimension. These cases are $c=2 b$, whence

$$
V(x, y, z)=V(x)+V(y)+V(z),
$$

so that the wavefunction $\Phi$ is separable as

$$
\Phi(x, y, z)=\Psi_{x}(x) \Psi_{y}(y) \Psi_{z}(z),
$$

and $c=0$, whence

$$
V(r, \theta, \phi)=V(r),
$$

so that

$$
\Phi(r, \theta, \phi)=R(r) Y_{l m}(\theta, \phi),
$$

where $Y_{l m}(\theta, \phi)$ is the standard spherical harmonic. The empirical parameters of Pitzer and Bernstein $a^{4} e^{4}$

$$
\begin{aligned}
& a=0.087 \pm 0.013 \mathrm{mdyn} / \AA, \\
& 0.05 \leq b \leq 0.075 \mathrm{mdyn} / \AA^{3},
\end{aligned}
$$

and

$$
0 \leq c \leq 0.08 \mathrm{mdyn} / \AA^{3} .
$$

One set of values obtained by fitting Wang and Lohr's crystal-field potential energy curves to the form (1) and (2) is

$$
\begin{aligned}
& a=0.062 \mathrm{mdyn} / \AA, b=0.029 \mathrm{mdyn} / \AA^{3}, \\
& \text { and } c=0.049 \mathrm{mdyn} / \AA^{3} ;
\end{aligned}
$$

other sets are described in the Results section. The above values of $a$ and $c$ are within the Pitzer-Bernstein range, but the value of $b$ is too small, reflecting an underestimated restoring force and an overestimated pseudo-Jahn-Teller displacement away from $O_{h}$ symmetry. Note that the assumption $c=2 b$ overestimates the angular barrier to pseudorotation corresponding to the parameters in (7) while the assumption $c=0$ corresponds to zero barrier. It is thus of interest to consider the band shape for an electronic transition from a ground state characterized by Devonshire parameters $a, b$, and $c$ to an excited state characterized by parameters $a^{\prime}, b^{\prime}$, and $c^{\prime}$, without restrictions being placed on these parameters.

\section{METHOD}

One approach to the band shape would be to expand the wavefunctions in a basis of three-dimensional HO functions in spherical polar coordinates. ${ }^{13}$ These basis functions may be written in the form of (6), so that the effect of $c \neq 0$ is to "mix" angular momentum states. However, Pitzer and Bernstein ${ }^{4}$ were successful in calculating the $t_{1 u}$ bending contribution to the entropy at $335{ }^{\circ} \mathrm{K}$ from the classical partition function

$$
Q(T)=h^{-3}(2 \pi \mu k T)^{3 / 2} \int e^{-V / k T} d \tau
$$

where $h$ is Planck's constant, $\mu$ is the reduced mass for the vibration, $k$ is the Boltzmann constant, $V$ is the Devonshire potential $(1,2), d \tau$ is the three-dimensional volume element, and the integration is taken over all space. The use of (9) is satisfactory at this $T$ in view of the close spacing of the quantum states. This may be seen quantitatively by considering a harmonic oscillator approximation at the $C_{3 v}$ radial minimum of the Devonshire potential. The $a$ in (7) corresponds to a $t_{1 u}$ bending frequency of $300 \mathrm{~cm}^{-1}$ using a reduced mass of $6.56 \mathrm{amu}$. The exact quantum mechanical density function for the harmonic oscillator has been given in closed form by Kubo and Toyozawa, ${ }^{14}$ and is Gaussian like the integrand in (9). The ratio of the classical Gaussian width parameter $\sigma$ to the exact quantum mechanical $\sigma$ is easily shown to be $[\tanh x) / x]^{1 / 2}$, where $x=h \nu / 2 k T$; this ratio is zero at $T=0$, but approaches unity as $T \rightarrow \infty$. Thus the classical width never exceeds the quantum mechanical width. For the above example the ratio of $\sigma^{\prime} \mathrm{s}$ is 0.938 at $335^{\circ} \mathrm{K}$ and 0.992 at $990^{\circ} \mathrm{K}$, showing the goodness of the classical approximations.

Detailed band shape studies have been made by Toyozawa and Ionue ${ }^{15}$ and by $\mathrm{Cho}^{16}$ for the electronic absorption of ions such as $\mathrm{Tl}^{+}, \mathrm{In}^{+}, \mathrm{Ga}^{+}, \mathrm{Sn}^{+2}$, etc., in alkali halide hosts. These ions are isovalent with $\mathrm{Xe}^{+6}$, so that the excitations considered are ${ }^{1} A_{1 \xi} \rightarrow{ }^{3} T_{1 u}$ and ${ }^{1} A_{1 g} \rightarrow{ }^{1} T_{1 u}$. Their main interest was in the effects of spin-orbit coupling and excited-state Jahn-Teller splittings, the latter arising from coupling with the $e_{g}$ stretching and $t_{2 g}$ bending modes of a hexahalo complex. The classical Condon approximation they used involves the classical partition function (9), and may be written

$$
I(E, T)=\frac{\int e^{-V / k T} \delta[E-\Delta V] d \tau}{\int e^{-V / k T} d \tau},
$$

where $V$ and $d \tau$ are in the appropriate multidimensional space, $I$ denotes the spectral intensity function, $E$ is the spectral energy, $\Delta V$ is the difference between excited and ground state potentials, and $\delta$ denotes the Dirac delta function. Factors of $(2 \pi \mu k T)^{1 / 2}$ and $h^{-1}$ for each vibration do not appear in (10) as they cancel in the quotient. In both the work of Toyozawa and Ionue ${ }^{15}$ and that of $\mathrm{Cho}^{16}$ the ground-state potential energy surface was taken as that for a multidimensional $\mathrm{HO}$, while that for the excited states had the equilibrium position displaced along the Jahn-Teller active coordinates. In some examples ${ }^{15}$ the band shape (10) was evaluated analytically, but in general ${ }^{16}$ it was evaluated numerically by the Monte Carlo integration method. Cho's application of the method was straightforward since pseudorandom numbers with a Gaussian distribution appropriate to a $\mathrm{HO}$ ground state are easily generated ${ }^{16}$ from random numbers. However, a different approach must be taken when $V$ in (10) represents the Devonshire potential $(1,2)$.

We employ a combination of rejection and inversion techniques as outlined by Fluendy. ${ }^{17}$ The rejection technique may be illustrated by a one-dimensional integrand

$$
f(x)=p(x) g(x),
$$


where the nonnegative factor $p(x)$ is viewed as a probability distribution function. We draw random $x_{i}$ 's from the range of $x$ considered. For each $x_{i}$ we choose as a "tag" another random number $f_{1}$ from the range zero to the maximum in $p(x)$. If $f_{i}>p\left(x_{i}\right)$, then $x_{i}$ is rejected, while if $f_{i} \leq p\left(x_{i}\right)$, then $x_{i}$ is accepted. Since the number of $x_{i}$ 's initially chosen near any point will be on the average the same as the number for any other point, then the number of $x_{i}$ 's accepted will be proportional to the height of $p(x)$ at this point. Thus the set of accepted $x_{i}$ 's will be distributed according to $p(x)$. The integral $I$ from $x_{1}$ to $x_{2}$ of $f(x)$ is then given by the estimator

$$
I=\frac{\left(x_{2}-x_{1}\right)}{N} \sum_{i=1}^{N} g\left(x_{i}\right),
$$

where the set of $N x_{i}$ 's have the nonuniform distribution $p(x)$. The inversion technique ${ }^{17}$ is an alternative way of obtaining the distribution $p(x)$ which depends upon knowing the inverse of the cumulative distribution function

$$
c d f\left(x_{i}\right)=\int_{x_{1}}^{x_{i}} p(x) d x .
$$

Uniformly distributed values of $y_{1}=c d f\left(x_{i}\right)$ then yield values of $x_{i}$ with the distribution $p(x)$.

For three dimensions we pick a random number for each variable and a fourth random number as the "tag" for comparison with the distribution function at the point. The cubic symmetry of (1) or (2) means that only a single octant need be considered so the four random numbers are chosen from the ranges

$$
\begin{aligned}
& 0 \leq A_{i} \leq A_{\max }, \\
& 0 \leq \theta_{i} \leq \pi / 2, \\
& 0 \leq \phi_{i} \leq \pi / 2,
\end{aligned}
$$

and

$$
0 \leq f_{i} \leq 1 ;
$$

the first is not the radius but instead an area the role of which is defined later in this section. Cartesian coordinates could be used, but we have estimated a greater efficiency with spherical polar coordinates provided that the anisotropy in $V$ is not too large.

The distribution function we use is

$$
p(r, \theta, \phi)=r^{2} \sin \theta \exp \left[-\left(V-V_{0}\right) / k T\right],
$$

in which the potential (2) has been shifted by the constant

$$
V_{0}=-3 a^{2} / 4(3 b-c) \text {, }
$$

where $V_{0}$ is the value of $V$ at its minimum which occurs along a (111) axis at

$$
r_{0}=[3 a / 2(3 b-c)]^{1 / 2}
$$

Thus $p(r, \theta, \phi)$ equals $r_{0}^{2}(2 / 3)^{1 / 2}$ at the minimum in $V$. The factor $r^{2} \sin \theta$ from the volume integration element is included in (15) to obtain a proper distribution of integration points; thus (15) is not simply the probability of the system being at a point $(r, \theta, \phi)$. Since $p(r, \theta, \phi)$ is sharply peaked for the temperatures and Devonshire parameters considered, a pure rejection technique in which the "tag" $f_{i}$ in (14) is compared to the ratio of

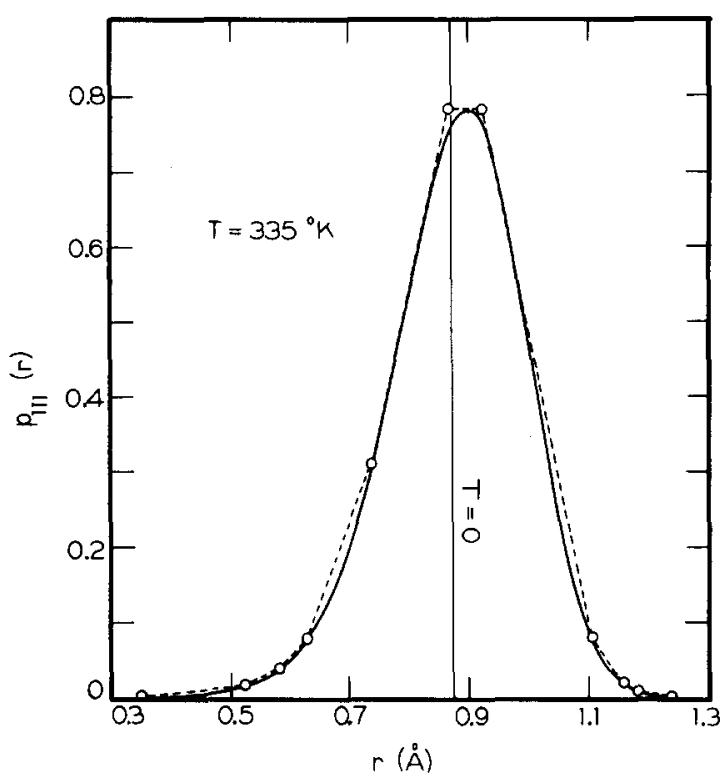

FIG. 2. Plots of the smooth bounding function $p_{111}(r)$ in (18) and the piecewise linear appropriation to it, $F(r)$, passing through the 11 circled common points for $T=335^{\circ} \mathrm{K}$ and Devonshire parameters (20). The vertical line at $r=r_{0}=0.87 \AA$ denotes the $T=0^{\circ} \mathrm{K}$ limit for $p_{111}(r)$.

$p(r, \theta, \phi)$ to the latter's maximum value would result in only a small percentage of random number sets being accepted. Instead we set up a bounding function ${ }^{16}$

$$
p_{111}(r)=r^{2} \exp \left\{-\left[-a r^{2}+(3 b-c) r^{4} / 3-V_{0}\right] / k T\right\},
$$

which is the value of (15) along a (111) axis divided by $(2 / 3)^{1 / 2}$. Since it is necessary that $p_{111}(r)$ be not less than $p(r, \theta, \phi)$ for all $\theta$ and $\phi$, the factor $\sin \theta$ is omitted from (18). This exclusion sets an upper limit equal to the mean value of $\sin \theta$, which is $2 / \pi$ or approximately 0.637 , to our acceptance fraction.

Radial endpoints $r_{\min }$ and $r_{\max }$ are now defined such that (18) is $10^{-3}$ of its maximum value. These endpoints do not correspond to the same value of the potential because of the $r^{2}$ factor in (18). We now approximate (18) by a piecewise linear bounding function $F(r)$ that can be readily integrated to yield

$$
A(r)=\int_{r_{\mathrm{min}}}^{r} F(r) d r ;
$$

the simple form of (19) is readily inverted to yield $r(A)$, the value of the radius corresponding to a given area. Thus from random values of $A_{i}(r)$ in the range zero to $A_{\max }$ we obtain pseudorandom numbers $r_{1}(A)$ in the range $r_{\min }$ to $r_{\max }$ and with the distribution $F(r)$ which closely matches the distribution (18).

Figure 2 shows a plot of $p_{111}(r)$ and the bounding function $F(r)$ at $T=335^{\circ} \mathrm{K}$ using the parameters

$$
\begin{aligned}
& a=0.087 \mathrm{mdyn} / \AA, \\
& b=0.064 \mathrm{mdyn} / \AA^{3},
\end{aligned}
$$

and

$$
c=0.020 \mathrm{mdyn} / \AA^{3} ;
$$




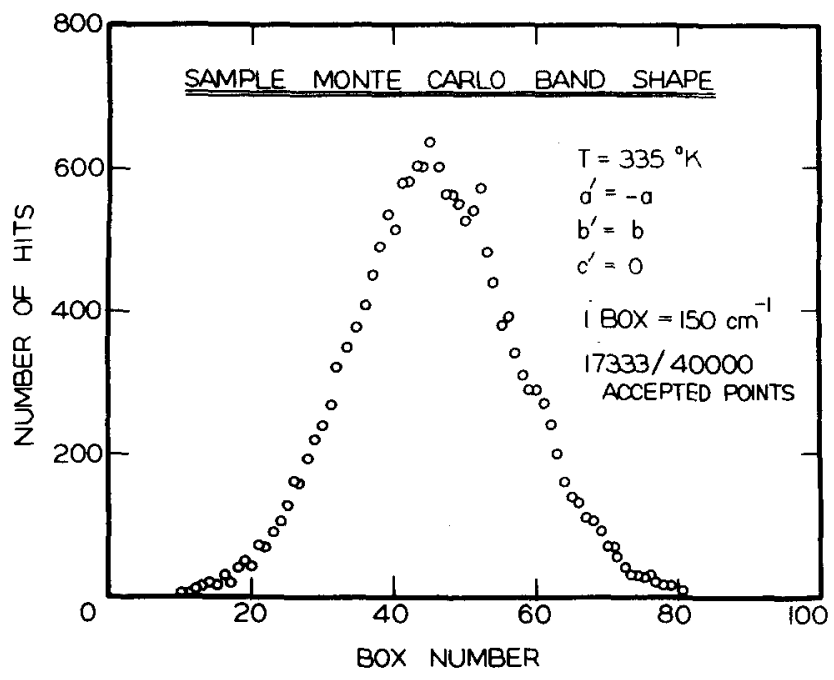

FIG. 3. Monte Carlo histogram for the example in Fig. 2 with excited-state Devonshire parameters $a^{\prime}=-a, b^{\prime}=b$, and $c^{\prime}=0$.

$a$ and $b$ are from the middle of the Pitzer-Bernstein estimates in (7), while $c$ is toward the lower end of its range. In this example $r_{\text {min }}=0.35 \AA$ and $r_{\text {max }}=1.25 \AA$. The solid vertical line in Fig. 2 denotes the value 0.87 $\AA$ for $r_{0}$ given by (17). The position $r_{m p}$ of the maximum in $p_{111}(r)$ increases linearly with $T$ in the limit of low $T$ as

$$
r_{m p} \cong r_{0}\left[1+(3 b-c) k T / 3 a^{2}\right] .
$$

We choose ten continuous linear segments for $F(r)$, including a horizontal segment at the maximum in $p_{111}(r)$, and then make a rejection test between the "tag" $f_{1}$ and the ratio $p(r, \theta, \phi) / F(r)$, leading to an acceptance rate of approximately $45 \%$ of the random number sets considered. This compares to a rate of approximately $7 \%$ for a pure rejection technique. If $V$ is isotropic $(c=0)$, we closely approach the $63.7 \%$ maximum acceptance rate determined by the mean value of $\sin \theta$.

The above procedure was written in FORTRAN IV for the Amdahl 470V/6 Computer at the University of Michigan and uses a standard uniform random number subroutine. For each accepted set $\left\{r_{i}, \theta_{i}, \phi_{i}\right\}$ we compute $\Delta V$ using Devonshire parameters for ground and excited states and assign a unit of intensity (a "hit") to the value $h \nu$ $=\Delta V\left(r_{i}, \theta_{i}, \phi_{i}\right)$. This step results from " $g(x)$ " in (11) being represented by the Dirac delta function $\delta[h \nu-\Delta V]$. The output is a histogram of the intensity distribution versus energy. The number of points necessary to achieve a reasonably smooth band shape was found to correspond to 17000 to 20000 pseudorandom number sets, meaning that about 40000 initial sets had to be generated. Figure 3 shows the results obtained at $335^{\circ} \mathrm{K}$ using the ground-state parameters (20) with excited state parameters $a^{\prime}=-a, b^{\prime}=b$, and $c^{\prime}=0$. This example is discussed in detail in the next section; here we note the acceptance of 17333 out of 40000 random numbers sets and the reasonably smooth band shape resulting from assigning "hits" to energy boxes of 150 $\mathrm{cm}^{-1}$ width. These boxes are sufficiently small since the fullwidth at half-height is $3750 \mathrm{~cm}^{-1}$. Hereafter we shall only show smooth curves drawn through points such as those in Fig. 3. As $T$ is increased $p(r, \theta, \phi)$, $p_{111}(r), F(r)$, and the band shape function all spread, so that a larger number of points is needed to achieve a smooth curve. The band shape function is readily normalized by a scaling inversely proportional to the number of accepted sets.

It should be noted that we have considered only the three $t_{1 u}$ bending modes in the evaluation of the band shape (10). The choice of Pitzer and Bernstein's empirical ground-state values of $a, b$, and $c$ does imply, however, the inclusion via parameterization of the leading terms in the coupling with other modes. The explicit dependence of the band shape on other modes would necessitate extending the dimensionality of the integrations in (10), a task readily borne, but at increased computation cost, by the Monte Carlo method. In particular the Condon distribution over the $a_{1 g}$ and the excited-state Jahn-Teller active $e_{g}$ and $t_{2 g}$ modes should be considered. A simple way of combining the results presented in the next section with those like Cho's in $a_{1 g}, e_{g}$, and $t_{2 g}$ spaces, is via the method of moments. This method was described by Wang and Lohr, ${ }^{8} 18$ and circumvents the evaluation of higherdimensioned integrals if the cubic and higher order coupling terms between the various modes are ignored so that $V$ is separable. For example the half-width at half-height for a band shape formed from the convolution of any number of Gaussians is proportional to the square root of the second moment and is hence given by the square-root of the sum of the squares of the halfwidths of the constituent Gaussians.

\section{RESULTS}

Although the Pitzer-Bernstein values of the Devonshire parameters provide a good description of the $\mathrm{XeF}_{6}$ electronic ground state, we lack comparable information for the electronic excited states. The crystalfield results ${ }^{6}$ in pure $t_{1 u}$ bending space indicated $O_{h}$ symmetry for the equilibrium geometry of the various excited states associated with the spin-orbit components of the ${ }^{3} P^{0}$ multiplet. A nonoctahedral geometry was predicted for the states arising from the ${ }^{1} P^{0} \mathrm{mul}-$ tiplet, but their distortions and pseudo-Jahn-Teller stabilizations are roughly only one-half of the corresponding ground-state values. The combination of spin-orbit and crystal-field splittings results in their being a total of eight potential energy curves for axially symmetric distortions of the states arising from the ${ }^{3} P^{0}$ and ${ }^{1} P^{0}$ multiplets, so that a detailed study is impractical. Instead we follow the example of the quantum mechanical study ${ }^{8}$ of the band shape, in that a simple representation of the excited state was assumed that closely resembled the crystal-field results. However, the quantum mechanical method used in that study restricted both ground- and excited-state potentials to the special cases $c=2 b(3,4)$ and $c=0(5,6)$. Table I lists the potentials used in that study together with their implied Devonshire parameters. The present studies are largely based on the empirical ground-state parameters (20) and several sets of assumed excitedstate parameters, the latter to be denoted by $a^{\prime}, b^{\prime}$, and $c^{\prime}$. 
TABLE I. Devonshire parameters from one-dimensional WangLohr potentials.

\begin{tabular}{lcll}
\hline \hline Potential $^{2}$ & $a(\operatorname{mdyn} / \AA)$ & $b\left(\operatorname{mdyn} / \AA^{3}\right)$ & $c\left(\operatorname{mdyn} / \AA^{3}\right)^{b}$ \\
\hline$-0.854 \xi^{2}+0.0124 \xi^{4}$ & 0.107 & 0.0772 & 0.1544 \\
$-0.854 \xi^{2}+0.0124 \xi^{4}$ & 0.107 & 0.0772 & $0^{c}$ \\
$-0.854 \xi^{2}+0.0372 \xi^{4}$ & 0.107 & 0.2316 & 0.4632 \\
$-0.722 \xi^{2}+0.00444 \xi^{4}$ & 0.0905 & 0.0276 & 0.0552 \\
$\xi^{2}$ & -0.125 & 0 & 0 \\
\hline \hline
\end{tabular}

${ }^{2}$ Reference $8 ; \xi$ is a dimensionless displacement variable.

${ }^{b}$ Except where assumed zero the $c$ parameter is constrained to equal $2 b$.

'Isotropy assumed; this potential was used in Ref. 8 in a radial rather than a Cartesian coordinate Schrödinger equation.

In order to test the Monte Carlo method we first considered the ground state defined by the parameters on line 1 of Table I. Since $c=2 b$, the potential is separable in Cartesian coordinates and a one-dimensional calculation can be made with a distribution function $p(x)$ similar to (15), but with the $r^{2} \sin \theta$ factor omitted. The excited-state potential is the parabola given by the parameters on line 5 of Table I.

Spectral moments are given by ${ }^{8,18}$

$$
\Lambda_{1}(T)=\bar{E}=\int \frac{I(E, T) E d E}{I(E, T) d E}
$$

and, for $k \neq 1$, by

$$
\Lambda_{k}(T)=\overline{(E-\bar{E})^{k}}=\int \frac{I(E, T)(E-\bar{E})^{k} d E}{I(E, T) d E},
$$

where $I(E, T)$ is given by (10). We compute (22) and (23) directly from the individual $p\left(X_{i}\right)$ and $\Delta V\left(X_{i}\right)$ values by appropriate summations rather than from the histograms. Calculations were made both for 300 and $600^{\circ} \mathrm{K}$ using random number sets of sizes $N=1000,2000$, 4000,8000 , and 16000 . In each case approximately $96 \%$ of sets were accepted, reflecting the goodness of the piecewise linear bounding function and the absence of a $\sin \theta$ factor in $p(x)$. Although the mean energy $\Lambda_{1}$ and the width parameter $\Lambda_{2}^{1 / 2}$ were found to be rather insensitive to the set size, the higher spectral moments were not. The skewness $S=\Lambda_{3} / \Lambda_{2}^{3 / 2}$ and the kurtosis $K=\left(\Lambda_{4} / \Lambda_{2}^{2}\right)-3$ did not stabilize until $N$ reached 16000 , where several repeated runs showed $S$ to be reproducible to approximately $27 \%$ and $K$ to approximately $10 \%$. For small $N$ a few "hits" with very large or small $\Delta E^{\prime}$ 's contribute excessively to the higher moments. Our subsequent calculations were all made with $N$ $=40000$, leading to 17000 to 20000 accepted sets as described in the Method section. The results of the present example are easily converted to three Cartesian dimensions by the relationships ${ }^{8} \Lambda_{1}^{\prime}=3 \Lambda_{1},\left(\Lambda^{\prime}\right)^{1 / 2}$ $=3^{1 / 2} \Lambda_{2}^{1 / 2}, S^{\prime}=S / 3^{1 / 2}$, and $K^{\prime}=K / 3$; these parameters are similar to the quantum mechanical results previously reported. Specifically, the shift in $\Lambda_{1}^{\prime}$, as $T$ increases from 300 to $600^{\circ} \mathrm{K}$ is here found to be 915 $\mathrm{cm}^{-1}$ as compared to $866 \mathrm{~cm}^{-1}$, the increase in $\left(\Lambda_{2}^{\prime}\right)^{1 / 2}$ to be from 1850 to $2850 \mathrm{~cm}^{-1}$ as compared to an increase from 2050 to $2930 \mathrm{~cm}^{-1}$, the change in $S^{\prime}$ to be from -0.30 to -0.44 as compared to a change from
-0.23 to -0.41 , and a change in $K^{\prime}$ from 0.05 to 0.13 as compared to a change from 0.12 to 0.14 . Negative $S$ corresponds to a skewness to low energy while positive $K$ corresponds to greater intensity in the "wings" than for a Gaussian with the same $\lambda_{2}$.

We considered next the excited-state potential defined by $a^{\prime}=-a, b^{\prime}=0$ and $c^{\prime}=0$, which is a threedimensional isotropic harmonic oscillator with an octahedral equilibrium geometry and a force constant of $2 a$, one-half of the ground-state value at the $r_{0}$ minimum. Figure 4 shows on a common energy scale the potentials used and the calculated band shapes at $T=225,550$, and $990^{\circ} \mathrm{K}$. In this and other examples the electronic trace is arbitrarily set equal to zero so that $V^{\prime}(r)=V(r)$ at $r=0$. The solid line marked $T=0$ denotes the value of $\Delta V \equiv V^{\prime}(r)-V(r)=3\left|V_{0}\right|$ at $r=r_{0}$, while there is an upper limit to $\Delta V$ of $4\left|V_{0}\right|$ at $r=2^{1 / 2} r_{0}$. This upper limit introduces a spectral cutoff as shown. If the excited-state force constant is increased, the cutoff shifts to higher energy. We also note that increasing $T$ causes the band maximum to shift to higher energy, but also causes the low energy tail to increase in intensity. Although the band for $T=335^{\circ} \mathrm{K}$ is not shown, its full-width at half-height would be approximately $2250 \mathrm{~cm}^{-1}$. For this example we find that the mean energy $\Lambda_{1}$ remains essentially constant as $T$ increases from 225 to $990^{\circ} \mathrm{K}$, but $\Lambda_{2}^{1 / 2}$ increases from 825 to $1220 \mathrm{~cm}^{-1}$, while $S$ goes from -0.65 to -0.96 and $K$ goes from 0.45 to 0.58 .

A related example is that shown in Fig. 5, with $a^{\prime}$ $=-a, b^{\prime}=b$, and $c^{\prime}=0$. The presence of the quartic term in $V^{\prime}$ that is not less than that in $V$ for any $\theta$ and $\phi$ assures that there is no upper limit to $\Delta V$ and hence no spectral cutoff. To a first approximation the band shape simply spreads from the $T=0$ line with increasing $T$, but there is some asymmetry and a slight shifting of the maximum to higher energy. The full-width at half-height for the $335^{\circ} \mathrm{K}$ band is approximately 4000 $\mathrm{cm}^{-1}$. For this example $\Lambda_{1}$ increases by $580 \mathrm{~cm}^{-1}$ as $T$ is increased from 335 to $990^{\circ} \mathrm{K}$, while $\Lambda_{2}^{1 / 2}$ increases from 1760 to $2280 \mathrm{~cm}^{-1}, S$ from -0.09 to +0.24 , and $K$ from -0.01 to -0.10 . The very small values of $S$ and $K$ at $335^{\circ} \mathrm{K}$ correspond to the band shape being nearly Gaussian, as seen in Fig. 3.

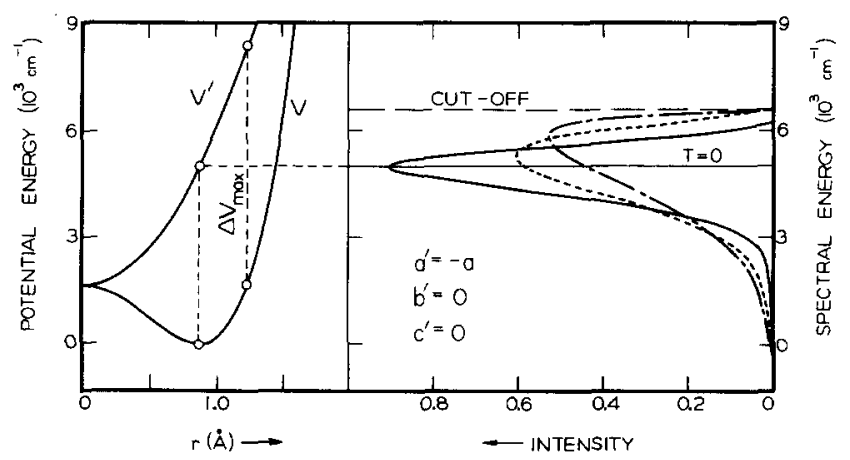

FIG. 4. Ground- and excited-state potential energy curves along a (11.1) axis together with Monte Carlo band shapes for the case $a^{\prime}=-a, b^{\prime}=0$, and $c^{\prime}=0$ at $T=225^{\circ} \mathrm{K}(\longrightarrow), 550^{\circ} \mathrm{K}$ $(-\cdots)$, and $990^{\circ} \mathrm{K}(\bullet-\rightarrow-\infty)$. 
The previous two examples incorporate the qualitative feature of an excited state with octahedral equilibrium geometry $(r=0)$. An example with a nonoctahedral geometry, such as that predicted in the crystal-field study ${ }^{16}$ for the states arising from ${ }^{1} P^{0}$, is in Fig. 6 . In this example $a^{\prime}=2 a, b^{\prime}=8 b$, and $c^{\prime}=0$. Again excitedstate isotropy is assumed, but with $a^{\prime}$ and $b^{\prime}$ chosen such that the excited-state minimum occurs at $r=(a)$ $8 b)^{1 / 2}$ with a stabilization of $-a^{2} / 8 b$, which is one-half of the ground-state stabilization along a (100) axis. The very steep rise of $V^{\prime}$ near the ground-state minimum at $r_{0}$ results in an extremely broad band shape; the full-width at half-height at $335^{\circ} \mathrm{K}$ is approximately $13100 \mathrm{~cm}^{-1}$ ! Here the band maximum shifts to lower energy with increasing $T$, but there is a low-energy cutoff $210 \mathrm{~cm}^{-1}$ below our arbitrary zero of energy. For this last example $\Lambda_{1}$ increases by $3290 \mathrm{~cm}^{-1}$ as $T$ is increased from 335 to $990^{\circ} \mathrm{K}$, while $\Lambda_{2}^{1 / 2}$ increases from 5970 to $11050 \mathrm{~cm}^{-1}, S$ from 0.86 to 1.24 , and $K$ from 0.89 to 1.92 , these various measures all reflecting the very large spread to higher energies shown in Fig. 6 .

\section{SUMMARY}

The spectral distribution with respect to the $t_{1 u}$ bending mode has been calculated by the Monte Carlo method for the ${ }^{1} A_{1 g}-{ }^{1} T_{1_{u}}$ transition in $\mathrm{XeF}_{6}$. A similar distribution, but with reduced intensity, is expected for the ${ }^{1} A_{1 g} \rightarrow{ }^{3} T_{1 \mu}$ transition. Pitzer and Bernstein's values ${ }^{4}$ of the Devonshire parameters are used to describe the ground state, while several sets of parameters are considered for the excited state. Previous applications ${ }^{15,16}$, of the Monte Carlo method to spectral band shapes have been confined to multidimensional harmonic oscillator ground-state potentials, thus only requiring random numbers with normal distributions. Our pseudorandom distributions are obtained easily by a combination of rejection and inversion techniques. The computation cost was approximately $\$ 4.00$ per run

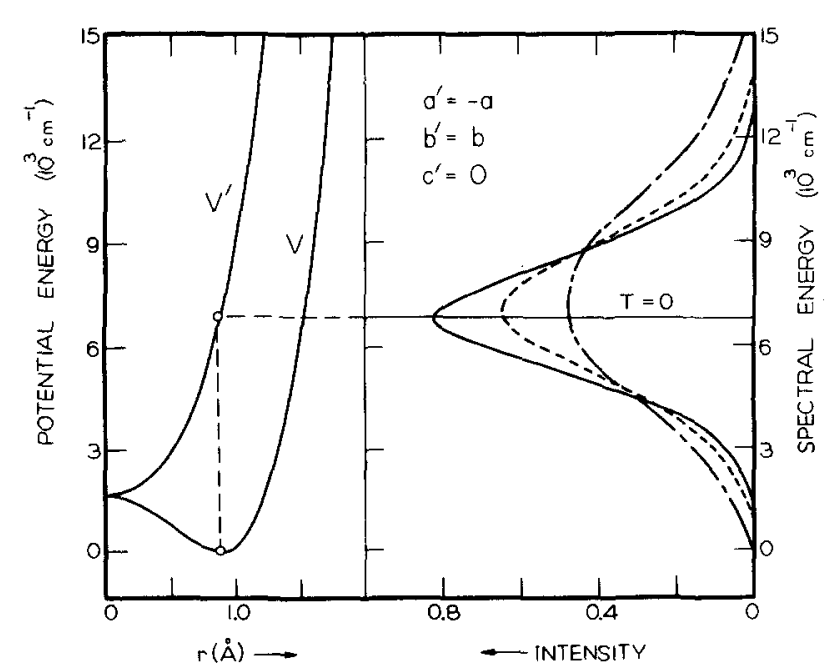

FIG. 5. Ground- and excited-state potential energy curves along a (111) axis together with Monte Carlo band shapes for the case $a^{\prime}=-a, b^{\prime}=b$, and $c^{\prime}=0$ at $T=335^{\circ} \mathrm{K}(\longrightarrow), 500^{\circ} \mathrm{K}$ $(---)$, and $990^{\circ} \mathrm{K}(\cdot-\cdots+-\infty)$.

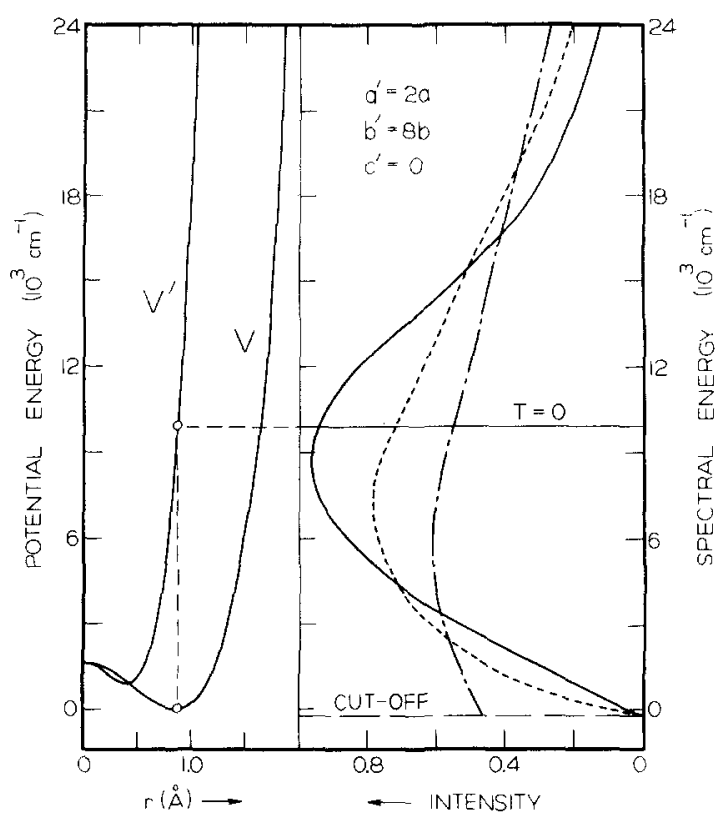

FIG. 6. Ground- and excited-state potential energy curves along a (1.1.) axis together with Monte Carlo band shapes for the case $a^{\prime}=2 a, b^{\prime}=8 b$, and $c^{\prime}=0$ at $T=335^{\circ} \mathrm{K}(\longrightarrow), 550^{\circ} \mathrm{K}$ $(----)$, and $990^{\circ} \mathrm{K}(\cdot-\cdots+-\infty)$.

on the Amdahl 470V/6 computer. Greater economy may be achieved by storing the pseudorandom numbers generated for a given temperature and ground-state potential and then computing band shapes for several different excited-state potentials. The resulting band shape consists of a single broad peak with a width very dependent on the choice of the excited-state parameters. The key difference between the present results and those in our earlier quantum mechanical study ${ }^{8}$ is the absence here of the shifts of the mean energy $\Lambda_{1}$ to lower energies as the temperature is raised. Note that the barrier to pseudorotation corresponding to the groundstate parameters we used (20) is only $47 \mathrm{~cm}^{-1}$ (the energy difference between the respective radial minima for $C_{2 v}$ and $C_{3 v}$ structures), which is small compared to the thermal energies considered, namely $k T=155$ to to $690 \mathrm{~cm}^{-1}$ for $T=225$ to $990{ }^{\circ} \mathrm{K}$. Thus there is considerable angular spread to the distribution (15). The $C_{4 v}$ and $O_{n}$ structures lie 173 and $1662 \mathrm{~cm}^{-1}$, respectively, above the $C_{3 v}$ minimum with this choice of parameters. By contrast the energy differences between the $C_{2 v}$ and $C_{3 v}$ minima of our previously used potentials ${ }^{8}$ were $1860,0,620$, and $3710 \mathrm{~cm}^{-1}$, respectively, for the four cases listed in Table I. When $c=2 b$, any angular spreading of the distribution (15) as $T$ is raised is necessarily accompanied by a shift to smaller $r$; this results from the fact that the radial coordinates of the $C_{4 v}, C_{2 v}$, and $C_{3 v}$ minima are in the ratios $1: 2^{1 / 2}: 3^{1 / 2}$. However for the parameters in $(20)$ the ratios are $1: 1.0415: 1.0565$, so that the $r^{2}$ factor in (15) greatly reduces the spreading to smaller $r$ and prevents there being a thermal red shift. (While we did find a red shift in our earlier calculation with $c=0$, that case was handled in a rigid pseudorotor approximation, thus not correctly accounting for centrifugal effects). 
The lack of much experimental detail (Fig. 1) with which to make comparisons makes the characterization of the excited states somewhat uncertain. There is structure in the $\mathrm{TeX}_{6}^{-2}$ spectra analogous to that seen for $s^{2}$ impurity ions in alkali halides, but any analogous structure appears "washed out" for $\mathrm{XeF}_{6}$. Both Toyozawa and Inoue ${ }^{15}$ and $\mathrm{Cho}^{16}$ showed that coupling of the ${ }^{1} T_{1 u}$ excited state with the $t_{2 g}$ mode produces a characteristic three-peak pattern for the ${ }^{1} A_{1 g} \rightarrow{ }^{1} T_{1 u}$ band shape. The logarithmic singularity of the central peak is removed by interactions with modes such as $a_{1 g}$ or $e_{g}$. Cho ${ }^{16}$ also discussed a three-peak pattern arising from coupling with the $e_{g}$ mode in the presence of spin-orbit coupling. This structure disappears as the ratio of the spin-orbit coupling coefficient to the $e_{g}$ Jahn-Teller coupling coefficient approaches zero or as the $a_{1 g}$ coupling coefficient becomes large. A similar washing out of these three peak patterns would result when the pseudo-Jahn-Teller coupling with the $t_{1 u}$ modes is large, as it is in $\mathrm{XeF}_{6}$. In conclusion we note a recent study ${ }^{19}$ in which a grouptheoretical analysis of rapid polytopal rearrangements of $\mathrm{XeF}_{6}$ was used to obtain splittings of the rigid molecule energy levels and to match the reported ir and Raman spectra.

\section{ACKNOWLEDGMENTS}

The authors wish to thank the University of Michigan Computing Center for use of the Amdahl 470V/6 Computer and Dr. L. S. Bernstein and Mr. E. K. Grimmelmann for numerous helpful discussions.

${ }^{1}$ A. F. Devonshire, Proc. Roy. Soc. (London) A 153, 601 (1936); for a review of studies of diatomic molecules in noble gas matrices and in ionic crystals see A. S. Barker,
Jr. and A. J. Sievers, Rev. Mod. Phys. 47, Suppl. No. 2 (1975).

${ }^{2}$ L. S. Bartell and R. M. Gavin, J. Chem. Phys. 48, 2466 (1968).

${ }^{3}$ R. M. Gavin and I. S. Bartell, J. Chem. Phys. 48, 2460 (1968).

${ }^{4}$ K. S. Pitzer and L. S. Bernstein, J. Chem. Phys. 63, 3849 (1975); also see L. S. Bernstein, Ph. D. thesis, University of California, Berkeley, May 1974 (Lawrence Berkeley Laboratory Report LBL-2701).

${ }^{5}$ F. Schreiner, D. W. Osborne, J. G. Malm, and G. N. McDonald, J. Chem. Phys. 51, 4838 (1969).

${ }^{6}$ S. Y. Wang and L. L. Lohr, Jr., J. Chem. Phys. 60, 3901 (1974); for erratum see J. Chem. Phys. 62, 2013 (1975).

${ }^{7}$ S. Y. Wang and L. L. Lohr, Jr. J. Chem. Phys, 60, 3916 (1974).

${ }^{8}$ S. Y. Wang and L. L. Lohr, Jr. J. Chem. Phys. 61, 4110 (1974).

${ }^{9}$ G. L. Goodman, J. Chem. Phys. 56, 5038 (1972).

${ }^{10} \mathrm{H}$. H. Claassen, G. L. Goodman, and H. Kim, J. Chem. Phys. 56, 5042 (1972).

${ }^{11}$ U. Nielsen, R. Hänsel, and W. H. E. Schwartz, J. Chem. Phys. 56, 5042 (1972).

${ }^{12}$ D. A. Couch, C. J. Wilkins, and H. Gray, J. Am. Chem. Soc. 92, 307 (1970).

${ }^{13}$ This method was used by I. S. Bernstein and K. S. Pitzer, J. Chem. Phys. 62, 2530 (1975), to obtain the "inversion" splittings for the electronic ground state.

${ }^{14} \mathrm{R}$. Kubo and Y. Toyozawa, Prog. Theor. Phys, (Japan) 13, 160 (1955); the expression is obtainable from the time-dependent Green's function by the substitution $t=-i \hbar / k T$.

${ }^{15}$ Y. Toyozawa and M. Inoue, J. Phys. Soc. (Japan) 21, 1663 (1966).

${ }^{16} \mathrm{~K}$. Cho, J. Phys. Soc. (Japan) 25, 1372 (1968).

${ }^{17}$ For a general description of the method see M. Fluendy, Markov Chains and Monte Carlo Calculations in Polymer Science edited by G. G. Lowry (Marcel Dekker, New York, 1970), pp. $45-90$.

${ }^{18}$ L. I. Lohr, Jr., J. Am. Chem. Soc., 92, 2210 (1970).

${ }^{19} \mathrm{C}$. Trindle, S. N. Datta, and T. D. Bouman, Int. J. Quantum Chem. (in press). 